\title{
Semantic-to-autobiographical memory priming occurs across multiple sources: Implications for autobiographical remembering
}

\author{
John H. Mace ${ }^{1} \cdot$ Merve Unlu ${ }^{1}$ \\ Published online: 2 March 2020 \\ (C) The Psychonomic Society, Inc. 2020
}

\begin{abstract}
Mace, McQueen, Hayslett, Stalely, and Welch (Memory \& Cognition, 47, 299-312, 2019) demonstrated that the activation of semantic memories leads to the activation of autobiographical memories. In that study, the semantic processing of concept words (e.g., garden) was shown to prime related autobiographical memories (e.g., personal memories involving garden) on voluntary and involuntary autobiographical memory tasks. Our goal in the current study was to replicate such semantic-to-autobiographical priming effects, and show that they can be extended to a wider set of stimuli than reported in Mace et al. In Experiment 1, semantic-to-autobiographical priming was obtained on a measure of involuntary autobiographical memory (the vigilance task) following the processing of concept words in insolation and within the context of a sentence. In Experiment 2, semantic-toautobiographical priming was again observed to occur with the vigilance task, but in this instance it occurred following the processing of both linguistic (words) and nonlinguistic (pictures) stimuli. The results of each of these experiments supports the idea that semantic-to-autobiographical activations occur within a wide variety of contexts (e.g., in language, perception, etc.). The implications of the results for autobiographical remembering are discussed.
\end{abstract}

Keywords Semantic-to-autobiographical priming $\cdot$ Autobiographical memory priming $\cdot$ Semantic priming $\cdot$ Autobiographical memory $\cdot$ Autobiographical memory functions $\cdot$ Semantic memory

\section{Introduction}

Throughout the course of a day, one processes massive amounts of information from many different sources (e.g., reading a newspaper or a book, watching television, engaging in conversation, etc.). Conway has argued that such routine general information processing continuously activates memories in the autobiographical knowledge base (e.g., Conway, 2001, 2005). Mace (2010) has also argued that activations between semantic and autobiographical memory are commonplace and that such semantic-to-autobiographical memory activations may play a considerable role in autobiographical remembering (e.g., everyday involuntary autobiographical remembering). In the present study, we investigated semanticto-autobiographical memory priming across multiple stimuli.

John H. Mace

jhmace@eiu.edu

1 Psychology Department, Eastern Illinois University, Charleston, IL 61920, USA
We follow up and extend a recent study (Mace, McQueen, Hayslett, Stalely, \& Welch, 2019) where semantic-toautobiographical memory priming was established. We outline the details of the current study below, as well as review the literature on semantic-to-autobiographical memory priming. However, we first outline our working model for semanticto-autobiographical memory priming.

\section{A general model of semantic-to-autobiographical memory activations}

Mace et al. (2019) argued that semantic access involves both a spread to related generic knowledge (e.g., as in semantic priming models, e.g., Anderson, 1983; Collins \& Loftus, 1975), as well as related autobiographical knowledge (see also Mace, 2010). To illustrate the autobiographical activations, reading or hearing the word Garden or School will activate a pool of autobiographical memories associated with each of these concepts (e.g., a memory of assisting your grandmother with gardening). These activations will consist of both general 
autobiographical memories (e.g., the knowledge that you use to assist your grandmother with gardening) and specific autobiographical memories (i.e., episodic memories, e.g., a memory for a specific instance of assisting your grandmother with gardening). Occasionally, these activations become conscious immediately and are experienced as involuntary memories (Conway, 2005; Mace, 2010). However, for the most part, autobiographical memory activations never surface into consciousness. The model also posits that because semantic-toautobiographical activations are constant, massive amounts of autobiographical memory activations will occur within the space of a day. Further, because of their massive numbers, and because the activations are likely to be long lasting (e.g., days; Mace, 2005), some of them will inevitably surface after their original processing episodes (minutes, hours, or days later) in response to overlapping internal or external cues where they then influence the production of voluntary or involuntary autobiographical memories (Mace et al., 2019).

To illustrate further with a specific example, imagine that one processes the concept $d o g$. In addition to all of the associated semantic features that become activated, this concept will activate a pool of related autobiographical memories. This pool will consist of a pool of general autobiographical memories (e.g., I had a dog when I was little; my sister has dogs; experientially based images of dogs, e.g., my dog; etc.), as well as a pool of specific autobiographical memories (i.e., episodic memories, e.g., taking my dog for a walk; playing with my sister's dogs, etc.). All of this activated knowledge, then, semantic and personal/autobiographical, informs the concept. As the autobiographical knowledge is likely to remain active for a significant period of time (up to days; Mace, 2005), it now has the potential of entering consciousness as involuntary autobiographical memories. For example, if one hears a dog bark or sees someone walking or playing with a dog, these cues could induce the spontaneous retrieval of any of the associated autobiographical memories (e.g., I had a dog when I was little; playing with my sister's dogs). It is possible that on occasion the priming process could lead to the production of additional unrelated memories. For example, if an episodic memory (e.g., playing with my sister's dogs) further activated content not related to the prime, but content within the memory (e.g., memories about one's sister), then such memories could also surface in future acts of autobiographical recall. While such memories would be distinguished from the prime by their incongruence with it, it is important to note that they, too, would emanate from the original priming event. It is also important to note that while it would be very difficult to establish these cases empirically, we mention them because semantic-to-autobiographical priming also has the potential of adding such memories to the processes of autobiographical recall.

\section{Semantic-to-autobiographical memory-priming studies}

Unlike implicit memory and semantic memory where a plethora of priming studies exist (McNamara, 2005; Roediger \& McDermott, 1993), only a small number of studies have investigated priming in autobiographical memory. Most of these studies investigated how autobiographical memories might prime other autobiographical memories (Ball \& Hennessey, 2009; Barzykowski \& Niedzwienska, 2018b; Conway \& Bekerian, 1987; Mace, 2005; Mace \& Clevinger, 2013). Of these studies, some have demonstrated that priming effects in autobiographical memory are long lasting (i.e., lasting minutes - Barzykowski \& Niedzwienska, 2018b; Mace \& Clevinger, 2013; or days - Mace, 2005). Of the total corpus of priming studies involving autobiographical memory, only three investigated how semantic memories might prime autobiographical memories (Conway \& Bekerian, 1987; Conway, 1990; Mace et al., 2019). In this group of studies, one failed to find priming between semantic and autobiographical memory (Conway \& Bekerian, 1987), one found priming under limited circumstances (Conway, 1990), and one found robust, relatively long-lasting semantic-to-autobiographical priming effects under a variety of circumstances (Mace et al., 2019).

In the first study to show semantic-to-autobiographical memory priming, Conway (1990) presented participants with taxonomic categories (i.e., category labels such as Birds or Vegetables) or goal-derived categories (i.e., category labels such as Birthday Presents or Camping Equipment). Following these priming phases, participants were given exemplar cues from each of the primed category labels (e.g., Sparrow or Potato for taxonomic categories, or Jewelry or Sleeping Bag for goal-derived categories) with the instruction to recall past personal experiences. Priming was then measured as retrieval time on exemplar cues in primed versus unprimed conditions. Overall, the results showed that goalderived categories had significantly shorter retrieval times relative to unprimed conditions, but no priming was evident for the taxonomic category conditions, though shorter latencies were consistently reported for this condition (Conway \& Bekerian, 1987, also failed to obtain priming with taxonomic categories). Thus, Conway's (1990) study suggested that semantic-to-autobiographical memory priming may occur under some, albeit limited, sets of circumstances.

More recently, Mace et al. (2019) argued that retrieval latencies may in some cases be a weak measure of semantic-toautobiographical priming, and that such priming might be more widespread and ubiquitous, as suggested in writings by Conway (2005) and Mace (2010). Thus, instead of retrieval latencies, Mace et al. (2019) measured semantic-toautobiographical priming more directly by observing the effect of primes (e.g., rating or reading the word music) on the content of subsequently produced autobiographical memories 
(i.e., observing if autobiographical memories concerned the content of primes, e.g., a personal memory involving music). Participants in the first experiment of the study rated words for the familiarity of their meanings (e.g., music, summer, quip, etc.) and then recalled autobiographical memories in response to unrelated word cues (e.g., flower). The results showed that primed participants produced significantly more autobiographical memories involving the content of the primes than unprimed participants. The results were replicated in Experiment 2 with an involuntary autobiographical memory task (i.e., the vigilance task; Schlagman \& Kvavilashvili, 2008), ${ }^{1}$ and in Experiment 3 where priming was observed following word-meaning familiarity ratings or lexical decision (e.g., decide if music, summer, quip, pulel are words). These results demonstrated semantic-to-autobiographical priming on different autobiographical memory measures (involuntary and voluntary tasks), and priming occurred following deep (familiarity ratings) and relatively more shallow processing conditions (lexical decision). Importantly, priming was obtained with a list of common words (e.g., music, sports, summer), including members of taxonomic categories (e.g., music, sports). Thus, in contrast to Conway (1990) and Conway and Bekerian (1987), the results of this study suggested that semantic-to-autobiographical priming is quite possibly ubiquitous.

\section{Implications of semantic-to-autobiographical memory priming}

Mace et al. (2019) argued that the implications of semantic-toautobiographical priming are considerable. For example, semantic-to-autobiographical priming is likely to affect voluntary and involuntary autobiographical remembering. In the case of voluntary remembering, such priming may facilitate or inhibit the recall of past experiences, depending on how prior semantic-to-autobiographical activations might coincide with intended autobiographical memory recall (i.e., facilitation when there is overlap in content, potential inhibition when content is opposing). An intriguing possibility here concerns the act of reminiscing (i.e., when individuals choose to recall certain periods of their lives, e.g., when they were young, in college, etc.; Fitzgerald, 1996; Mace, 2005; Mace \& Clevinger, 2013). When individuals engage in this process, some (or much) of what they "choose" to remember may be influenced by the unconscious forces of semantic-toautobiographical priming, making the process, in part, involuntary. Relatedly, everyday involuntary autobiographical remembering is also likely to be affected by semantic-to-

\footnotetext{
${ }^{1}$ The vigilance task elicits involuntary autobiographical memories by presenting participants with slides containing horizontal or vertical lines with embedded verbal cues. The embedded cues have been shown to occasionally elicit spontaneous memories and thoughts throughout the task (e.g., Schlagman \& Kvavilashvili, 2008).
}

autobiographical priming (Berntsen, 2009; Mace, 2007, 2018). Here, Mace et al. (2019) argued that the content of everyday involuntary autobiographical memories may be massively influenced by semantic-to-autobiographical priming, as illustrated earlier.

\section{Overview of study}

Our goal in the present study was to obtain further support for semantic-to-autobiographical memory priming. In Mace et al. (2019), semantic-to-autobiographical priming was demonstrated following the processing of a list of individually presented words. Here, we set out to demonstrate that such priming is more widespread by showing that it occurs with different forms of linguistic stimuli, and with nonlinguistic stimuli. We present two experiments that examined semantic-toautobiographical priming with linguistic stimuli (sentences or words, Experiment 1), and nonlinguistic stimuli (pictures, Experiment 2). In both Experiments we used an involuntary autobiographical memory task (the vigilance task) as the measure of priming.

The theoretical motivation behind these experiments was straightforward. If multiple processing sources cause semantic-to-autobiographical activations, then such activations may play a large role in autobiographical memory production, namely, the production of involuntary autobiographical memories. That is, if massive amounts of autobiographical memory are activated routinely, then occasionally such memories should come to mind as involuntary memories either immediately or sometime later - minutes, hours, or days (Conway, 2005; Mace, 2005, 2010), as discussed earlier. As noted earlier, the results of the second experiment in Mace et al. (2019) supported the notion that semantic-toautobiographical priming is a source of everyday involuntary memories. We therefore combined the vigilance task with priming from multiple stimuli because this would add more power to the claim that much everyday involuntary remembering is delayed semantic-to-autobiographical activations, or the byproducts of this process. Such an inference may help to explain the common phenomenon of involuntary remembering, as it provides clues about the potential functions of the phenomenon, or malfunctions if they are merely cognitive failures (we discuss this further in the General discussion).

Concerning predictions, generally, we predicted that semantic-to-autobiographical priming would manifest as an increase in the proportions of autobiographical memories related to the primes in the priming groups relative to the controls. Consistent with prior observations (e.g., Mace, 2005; Mace \& Clevinger, 2013; Mace et al., 2019), we did not expect the priming groups to have more memories overall than control, only more memories associated with the primed content. This expectation is consistent with the fundamental theory of priming in autobiographical memory, which states that 
priming influences the content of involuntary autobiographical memories (e.g., Berntsen, 2009; Mace, 2005; Mace \& Clevinger, 2013; Mace et al., 2019). While we believe that priming also influences involuntary memory production frequency, we also believe it would be exceedingly difficult to measure this aspect of it, as one may not be able to obtain an adequate baseline comparison condition (i.e., ideally, a group that has not been primed by everyday experience).

\section{Experiment 1}

In Experiment 1, we used the vigilance task to assess semantic-to-autobiographical priming following the processing of words or sentences. Participants in the word-priming group were given a list of words (e.g., music) for which they had to decide if the words had sensible meanings (e.g., music, quip, lotely). These words were embedded in sentences for the sentence-priming group for which participants in that group similarly decided if sentences had sensible meanings (e.g., people enjoy listening to music; their outfits that day were very quip). To assess the influence of priming on autobiographical memories, participants were subsequently engaged in the vigilance task (Schlagman \& Kvavilashvili, 2008) (control participants were also employed in this task). As noted, the vigilance task is a method for measuring involuntary autobiographical memories. (e.g., Barzykowski \& Staugaard, 2016; Mace et al., 2019; Schlagman \& Kvavilashvili; Barzykowski \& Niedzwienska, 2018b; Vannucci, Pelagatti, Hanczakowski, Mazzoni, \& Paccani, 2015). In this task, participants are presented with a large number of slides containing either vertical or horizontal lines. Embedded within each slide is a simple phrase (e.g., drinking from a cup), which participants are told to ignore. Participants are also told that they might experience spontaneous thoughts or memories during the task, and if so, they are to report them.

In Mace et al. (2019), semantic-to-autobiographical priming was found following the processing of words in isolation. As such, the results from that study potentially limit the conclusions to situations where words are processed in isolation. However, the semantic-to-autobiographical priming hypothesis is more expansive than this, as it asserts that semantic-toautobiographical activations occur in a wide variety of contexts with a wide variety of stimuli. Thus, whenever concepts are processed, their autobiographical referents are activated, and semantic-to-autobiographical priming occurs no matter the context in which they are processed (i.e., seen in isolation, as a part of a sentence, etc.). Accordingly, in this experiment we predicted that semantic-to-autobiographical priming would occur in both the word-priming and sentence-priming groups, and that priming would not differ across the groups. We reasoned that such results would allow one to assert that semantic-to-autobiographical priming occurs across a wide variety of linguistic contexts (e.g., reading words, sentences, or larger passages, listening or engaging conversation, etc.).

\section{Method}

\section{Participants}

The participants were 64 undergraduate students from Eastern Illinois University, who participated in exchange for course credit. Twenty-two of the participants served in the control group, and 21 in served in each of the priming groups. Thirty-nine of the participants were females, and 25 were males, with an age range of $18-22$ years $(M=19.1$ years).

\section{Materials}

The stimuli used in the priming phase for the word-priming group consisted of 38 relatively high- (e.g., music) and lowfrequency (e.g., quip) words, and non-words (e.g., lotely) (see Appendix 1), printed and presented in booklet form. The highfrequency words had frequency counts in the Corpus of Contemporary American English ranging from 6,149$356,731(m=81,836)$, while the low-frequency words had a range of 77-679 $(m=385)$. As in Mace et al. (2019), we were not interested in manipulating word frequency, nor did we expect semantic-to-autobiographical priming to occur with low-frequency words. The low-frequency words (and nonwords) were used merely to legitimize the cover story. The stimuli used in the priming phase for the sentence-priming group were constructed from the 38 stimuli used in the word-priming group list (also printed and presented in booklet form). Here, the words (and non-words) were used to construct 38 sentences (e.g., people enjoy listening to music; their outfits that day were very quip). The sentences were mostly single clauses, and many of them contained additional concepts that were not presented to word-priming participants (e.g., it rained throughout Mary's entire vacation, where vacation was a target concept word in the word-priming group and rained was an additional concept not presented in the word-priming group; see Appendix 2). The additional concepts were not intended, but occurred as a function of having relatively complex sentences, as we did not wish to only use simple sentences (e.g., Jim likes sports). Nevertheless, we used the additional concepts for a secondary priming assessment, where priming on the additional items was assessed against the control and word-priming groups. We refer to the set appearing in both priming groups as primary targets, and the additional set appearing in the sentence group alone as secondary targets.

Stimuli for the vigilance task consisted of 512 slides containing either horizontal or vertical lines, each with a unique word phrase (e.g., going to work) embedded in the center of the slide. 477 of the slides contained horizontal lines, 35 
contained vertical lines. The slides were presented randomly for $1.5 \mathrm{~s}$ on a computer screen, via the SuperLab (version 4.5) software, for a total duration of approximately $13 \mathrm{~min}$. Some of the embedded phrases contained the primary target words used in the sentence- and word-priming groups (22, e.g., friend or pet, with a few appearing in more than one phrase), some contained the secondary target items (13, e.g., work, also with a few appearing more than once), some could be seen as associated with the target concepts (43 in total, e.g., getting $a$ $\underline{d o g}$ ), while most (422) appeared to be unrelated (e.g., reaching for the stars).

The design of the vigilance task matched Schlagman and Kvavilashvili's (2008), with some minor variations. For example, we used 512 slides instead of the 600-800; 35 instead of 11-15 contained vertical lines; and the slides were presented randomly rather than in a fixed order. A more substantial variation included instructions on task-unrelated thoughts. In the current study (as well as in Mace et al., 2019), we asked participants to report both task-unrelated thoughts and involuntary memories (see below), while Schlagman and Kvavilashvili (2008) asked participants to only report involuntary memories.

\section{Procedure}

All participants were tested individually. Sentence- and wordpriming group participants were first engaged in their respective priming task, followed by the vigilance task. Each task was presented to participants as a separate study. The time lag between the priming task and the memory task was approximately $5 \mathrm{~min}$ on average. This was merely the average time between these tasks. Control group participants were only engaged in the vigilance task. In the word-priming task, participants were told that they were being involved in a study on word meanings. They were instructed to read the words printed in the booklet and decide if they had a sensible meaning, answering "yes" or "no" by circling one of the two responses printed next to each of the words. In the sentencepriming task, participants were told that they were being involved in a study on sentence meanings. They were instructed to read the sentences printed in the booklet and decide if they had a sensible meaning, answering "yes" or "no" by circling one of the two responses printed next to each of the sentences. Each group worked through the priming stimuli at their own pace. In the vigilance task, participants were told that they were being involved in a study on concentration. They were told they would see slides with either horizontal or vertical lines, and they were to say "yes" out loud whenever the slides contained vertical lines. They were further told that the slides would also contain phrases, but they were to ignore them. To ensure their understanding of the instructions, they then received four practice slides, one containing vertical lines. Once it was clear that they understood, they were further instructed that it was possible that they may experience taskunrelated spontaneous thoughts or memories (the concept of involuntary memories was explained to them), and if they experienced one or the other, they were to click the mouse and record them in a booklet that contained sheets of lined paper. Once this aspect was understood, the task commenced. On trials where participants clicked the mouse, an instruction screen would pop up reminding them to record their thoughts or memories in the booklet, clicking the mouse again when finished to return to the vigilance program. Following the vigilance task, all participants were asked to read through their booklet, marking entries as either spontaneous thoughts or memories. They were further instructed on the differences between general (i.e., more abstract autobiographical memories, such as "I went to London in 2005") and specific autobiographical memories (i.e., episodic memories, such as remembering "having a flat tire while driving across the Tower Bridge"), and they were asked to mark their memories as general or specific.

\section{Categorization method}

The content of the autobiographical memories for both the control and priming groups was read by two independent judges. The judges were looking for memories that clearly were personal memories involving the concepts presented in the priming phase. For example, personal memories involving, car, music, summer, hiking, and so forth (e.g., I remember listening to music in the park, or I remember hiking in the mountains). Memories were deemed as primed (conceptually overlapping) memories only in cases where the content of the memory clearly involved the content of items from the priming list, as in the examples given. Memories were deemed primed if they involved the explicit (e.g., I remember when I was listening to music with ... .) or implicit (e.g., I remember when I was listening to Beethoven's 5th with ... .) description of a concept, as conceptual overlap was the critical factor. In no event were memories deemed primed memories if words were used to simply describe aspects of an event (e.g., I remember the teacher quipped ......), eliminating possible instances of repetition priming, or if they were used as tangential references (e.g., I remember we spent three weeks in Florida, visiting Disney Land, ... , and I think we traveled by car on that trip). In cases where memories contained overlapping content from two (or more) of the target items, the memories were only counted once in the scoring process. The coding was done with both primary and secondary targets. Disagreements between the judges were settled through discussion. The Kappa statistic indicated high concordance between the judges on the categorizations, $K=.86, S E=$ .02 , primary targets; $K=.89, S E=.02$, secondary targets. 


\section{Results and discussion}

An examination of the total number of spontaneous memories and thoughts reported on the vigilance task showed that the word-priming group reported an average of 15.27 ( $S D=$ $12.63)$ memories and $3.55(S D=7.34)$ thoughts on the vigilance task, the sentence-priming group reported an average of $16.38(S D=15.95)$ memories and $2.98(S D=6.90)$ thoughts, whereas the control group reported an average of 11.13 ( $S D=$ $6.83)$ memories and $3.18(S D=7.77)$ thoughts. Seven participants reported no memories (one in each priming group and five in control). Two independent-samples analysis of variance (ANOVA) tests (one conducted on the memories, the other on thoughts) found no significant differences between the groups in either the total memories or thoughts reported $(F \mathrm{~s}<1.0)$. Of the memories reported, $68-72 \%$ were specific memories (72\%, control group; 68\%, word-priming group; $71 \%$, sentence-priming group, $\left.\chi^{2}(2)<1.0\right)$. Thus, these results show that the groups did not differ fundamentally in total number of thoughts or memories generated or in the generation of general or specific autobiographical memories.

Turning to the main question of interest, we analyzed both the primary (i.e., target concepts presented to both the word and sentence groups) and secondary (i.e., additional concepts produced by the sentences) target concepts for semantic-toautobiographical memory priming. The results of these analyses are presented in Table 1. The results represent the proportion of autobiographical memories (both specific and general memories) found to involve the primary or secondary concepts presented in the priming phases for the word and sentence groups. Concerning the primary targets, as can be seen in the table (top panel), both the word- and the sentence-priming groups produced more memories involving the primary targets than the control group. These results were subjected to a one-way independent-samples ANOVA, which indicated a significant effect of semantic-to-autobiographical priming, $F(2,62)=3.77, M S E=0.04, p<.05, \eta_{\mathrm{p}}^{2}=.11$. Follow-up with Fisher's least significant difference (LSD)

Table 1. Mean proportions of autobiographical memories (both specific and general) involving primary and secondary concepts in the control, sentence- and word-priming groups

\begin{tabular}{llll}
\hline Primary concepts & & & \\
Group & Control & Sentence & Word \\
Mean & .28 & .44 & .44 \\
SD & .24 & .24 & .17 \\
Secondary concepts & & & \\
Group & Control & Sentence & Word* \\
Mean & .01 & .07 & .01 \\
SD & .02 & .07 & .02 \\
\hline
\end{tabular}

*The word group also served as a baseline control in the secondary concept analysis statistic showed both priming groups differing from the control, but not from one another $(L S D=.13)$. Concerning the secondary targets, in this analysis both the word-priming and the control group served as baseline references, given that neither experienced these primes before testing on the vigilance task. As can be seen in the Table 1 (bottom panel), both the word-priming and the control groups produced fewer memories involving the secondary targets than the sentence group. These results were subjected to a one-way independent-samples ANOVA, which also indicated a significant effect of priming, $F(2,62)=13.62, M S E=0.002, p<.001, \eta_{\mathrm{p}}{ }^{2}=$ .31. Follow-up with the LSD statistic, not surprisingly, showed that the sentence group differed significantly from the word and control groups, while the latter two did not differ from one another $(L S D=.03)$. Because the computation of proportions can produce extreme scores (e.g., 1.0), we examined the data for extreme scores. This inspection showed that only one such extreme score existed (in control), and removal of that score did not change the statistical outcome.

Further analysis of the results with the primary targets showed that like in Mace et al. (2019), priming on the primary target set was obtained only with the high-frequency words, with a significant number of different targets appearing in the data overall ( 22 out of 26 high-frequency words). An analysis of the secondary targets showed that nine of the 38 targets in that set were represented in the data. Finally, at debriefing, participants indicated they had no knowledge of the research hypothesis or the underlying purpose of the vigilance task, thus ruling out the possibility that the results were due to deliberate recall of memories associated with the target items, or awareness of the research hypothesis.

In summary, the results of this experiment replicated the semantic-to-autobiographical priming effects reported in Mace et al. (2019). As in that study, we found semantic-toautobiographical priming effects following the processing of words presented in isolation (word-priming group). However, with the addition of a sentence-priming group, this study found semantic-to-autobiographical priming for these words when they were presented within the context of a sentence. In addition, we also found semantic-to-autobiographical priming in the sentence group for the additional concept words (e.g., work) that were presented in the sentences. These results suggest that autobiographical activations are not limited to special processing circumstances (e.g., scenarios where words are processed in isolation), but routinely occur in all forms of linguistic processing (e.g., reading words in isolation, in sentences or longer passages, engaging in conversation, etc.). Additionally, because the results were obtained with the vigilance task (a common measure of involuntary autobiographical memory), they also support and expand the claim that semantic-to-autobiographical priming plays a role in the production of involuntary autobiographical memories. We defer discussion of this point to the General discussion. 


\section{Experiment 2}

The semantic-to-autobiographical memory activation hypothesis states that whenever concepts are processed, the autobiographical memories associated with these concepts become activated. Thus, as semantic access is not limited to language processing, semantic-to-autobiographical activations should also occur outside of the language domain. Our goal in Experiment 2 was to gain support for this general claim by showing that semantic-to-autobiographical priming occurs with non-linguistic stimuli (pictures). To test this, we compared semantic-to-autobiographical priming following word processing (as in Experiment 1) and picture processing (pictorial images of target concept words), where we predicted that semantic-to-autobiographical priming would be found with both sets of stimuli. The experiment consisted of separate word- and picture-priming groups, along with a control condition, and priming was once again assessed with the vigilance task.

\section{Method}

\section{Participants}

The participants were 62 undergraduate students from Eastern Illinois University, who participated in exchange for course credit. Twenty of the participants served in the picturepriming group, and 21 each in the word-priming and control groups. Forty of the participants were females, and 22 were males, with an age range of $18-26$ years $(M=19.5$ years $)$.

\section{Materials}

The stimuli and design for the word-priming group and vigilance task were the same as in Experiment 1. Stimuli for the picture-priming group consisted of 38 color images. The images were either photographs (26), an icon (1), or abstract images $(11$ - i.e., various abstract forms, e.g., fragments of geometrical forms), all selected for representing (or replacing) the concepts or non-words used for the word-priming group. The abstract images were used in place of the low-frequency words and non-words (Appendix 1), and none had any obvious meaning. Each of the photo images was the photographic representation of the high-frequency concept words (e.g., a photo of a car; see Appendix 1 for the list of high-frequency words). Most of these clearly represented the concept (e.g., car, mountain, beach, etc.), some may have been interpreted in more than one way (e.g., the picture for home could also be interpreted as house, for city as buildings), while a few were more suggestive of the concept (i.e., friends, summer, and internet, where friends was represented by a group of young people appearing to have fun, summer by an image suggestive of summer, internet by the Microsoft Explorer icon), and could also be interpreted in more than one way. The images were public domain images extracted from Google Images. They were presented individually in Microsoft PowerPoint slides, all of the same approximate size (roughly $3.5 \mathrm{in.} \times$ 6.0 in.), and each in a plain, white background slide. Appearing in the top left-hand corner of each slide was the slide number, beginning with number 1 and ending with 38 .

\section{Procedure}

All participants were tested individually. The procedures for the vigilance task, word-priming, and control groups were identical to Experiment 1. For the picture-priming group, participants were first engaged in the picture-priming task, followed by the vigilance task, with approximately 5 min of lag time between tasks. In the picture-priming phase, participants were told that they were being involved in a study on image meanings. They were instructed to view each image and decide if they had a sensible meaning, answering "yes" or "no" by circling one of the two responses printed on an answer sheet that was numbered 1-38 with the words "yes" and "no" printed in each numbered space. The pictures were presented on a computer screen via Microsoft PowerPoint. As with the word-priming group, participants worked through the priming stimuli at their own pace. All other aspects of the experiment for this group (i.e., vigilance task and post-test phases) were identical to the word and control groups.

\section{Categorization method}

Using the same criteria specified for Experiment 1, the judges examined the memories of the priming groups and the control group for content overlap. The coding was done based solely on the verbal items used for the word-priming group (Appendix 1). The Kappa statistic indicated high concordance between the judges, $K=.89, S E=.01$.

\section{Results and discussion}

An examination of the total number of spontaneous memories and thoughts reported on the vigilance task showed that the word-priming group reported an average of $16.42(S D=$ $14.56)$ memories and $3.28(S D=7.39)$ thoughts, the picturepriming group reported an average of $15.60(S D=14.32)$ memories and $3.15(S D=7.47)$ thoughts, whereas the control group reported an average of $13.67(S D=10.56)$ memories and $3.42(S D=6.11)$ thoughts. Three participants reported no memories (one in the word-priming group and two in the control group). Two independent-samples ANOVAs (one conducted on the memories, the other on thoughts) found no significance between the groups in either the memories or the thoughts reported $(F \mathrm{~s}<1.0)$. Of the memories reported, $68-70 \%$ were specific memories $(68 \%$, control and word- 
priming groups; $70 \%$, sentence-priming group, $\left.\chi^{2}(2)<1.0\right)$. Thus, as in Experiment 1, these results show that the groups did not differ fundamentally in total number of thoughts or memories generated or in the generation of general or specific autobiographical memories.

Turning to the main findings, the results for the three groups are presented in Table 2. As can be seen in the table, the word- and picture-priming groups had more autobiographical memories corresponding to the concepts presented in their priming phases than the control group. These results were subjected to a one-way independent-samples ANOVA, which confirmed a significant effect of semantic-to-autobiographical priming, $F(2,59)=7.74, M S E=0.03, p=.001, \eta_{\mathrm{p}}{ }^{2}=.21$. Follow-up with the Fisher LSD statistic showed both priming groups differing from the control, but not from one another $(L S D=.11)$. An examination of the data for extreme scores showed that no such scores had existed among the three groups.

Further analyses showed that priming was only found with the target high-frequency concepts in both priming groups (as in Experiment 1). Also as in Experiment 1, a high number of the targets were represented in the overall data (21 out of 26 high-frequency words or pictures). Finally, at debriefing, participants indicated they had no knowledge of the research hypothesis or the underlying purpose of the vigilance task, thus ruling out the possibility that the results were due to deliberate recall of memories associated with the target items.

In summary, the results of this experiment replicated the semantic-to-autobiographical priming effects reported in Mace et al. (2019) and Experiment 1 of this study. Importantly, however, semantic-to-autobiographical priming was found following the processing of images, as well as their linguistic counterparts, and priming did not differ between these processing conditions. These results show that semantic-to-autobiographical priming is not limited to linguistic stimuli and linguistic processing. They suggest that semantic-to-autobiographical activations routinely occur across a wide set of circumstances (e.g., language processing, visual perception, etc.). Thus, they support the general claim of the semantic-to-autobiographical memory activation hypothesis, which states that whenever concepts are activated, the autobiographical memories associated with these concepts become activated.

Table 2. Mean proportions of autobiographical memories (both specific and general) involving concepts in the control, picture- and word-priming groups

\begin{tabular}{llll}
\hline Group & Control & Picture & Word \\
\hline Mean & .28 & .49 & .45 \\
SD & .20 & .16 & .18 \\
\hline
\end{tabular}

\section{General discussion}

Mace et al. (2019) demonstrated that the activation of semantic memories leads to the activation of autobiographical memories. Our goal in the current study was to replicate these findings and show that they could be extended to a wider set of stimuli than reported in Mace et al. (2019). The two experiments reported here each accomplished this objective. In Experiment 1, semantic-to-autobiographical priming was obtained on the vigilance task following the processing of concept words in insolation and within the context of a sentence. In Experiment 2, semantic-to-autobiographical priming was again observed to occur with the vigilance task, but this time it occurred following the processing of both linguistic (words) and nonlinguistic (pictures) stimuli. The results of each of these experiments supports the idea that semantic-toautobiographical activations occur within a wide variety of contexts (e.g., in language, perception, etc.).

Taken together, the results of Experiments 1 and 2 support the idea that semantic-to-autobiographical activations result from many different sources (reading, listening, perceiving, etc.). The results also support the idea that such semantic-toautobiographical priming will affect involuntary and voluntary retrieval processes in autobiographical memory. How much and to what extent each of these retrieval processes is affected depends on the time course of semantic-to-autobiographical priming. The data presented here, and in Mace et al. (2019), suggest that semantic-to-autobiographical priming lasts at least several minutes. While studies of semantic priming have shown such effects to last for both long (e.g., Coane \& Balota, 2009) and short (e.g., McNamara, 1992) intervals, studies of autobiographical priming have shown that autobiographical priming is consistently long-lasting (Barzykowski \& Niedzwienska, 2018b; Mace, 2005; Mace \& Clevinger, 2013), with durations up to several days (Mace, 2005). Thus, given the amount of semantic information that one processes in the space of minutes, hours, and days, we presume that semantic-toautobiographical priming results in massive amounts of longlasting autobiographical activations, all which have the potential of influencing involuntary and voluntary autobiographical remembering. The effects and implications of such activations on involuntary and voluntary of autobiographical memories appear to be asymmetrical.

For voluntary retrieval, it is difficult to gauge how semanticto-autobiographical priming might facilitate or inhibit the recall of sought after autobiographical memories (e.g., trying to remember if you ever went to a Malaysian restaurant; see further discussion in Mace et al., 2019). However, one thing that the data presented here, and in Mace et al. (2019), seems to suggest is that semantic-to-autobiographical priming should influence reminiscence recall. In reminiscence recall, one seeks to generally recall a particular time of life (e.g., when one was a child, in high school, etc.; e.g., Fitzgerald, 1996; Mace, 2005; 
Mace \& Clevinger, 2013). As one may often reminiscence without seeking particular targets, we believe that the memories that come to mind during this process may often be a product of prior semantic-to-autobiographical priming (for supporting findings, see Mace et al., 2019, Experiments 1 and 3). As noted at the outset, this is intriguing because it suggests that a voluntary process is not so voluntary, as the unconscious influences of semantic-to-autobiographical priming in part determines what is remembered. Future studies can address this more directly by manipulating semantic-to-autobiographical priming along with memory measures that closely mimic reminiscing.

The implications of semantic-to-autobiographical priming for involuntary autobiographical remembering are quite different. In Experiments 1 and 2, and in Mace et al. (2019, Experiment 2), nearly one-half of the involuntary memories produced on the vigilance task in primed conditions were a function of semantic-to-autobiographical priming. We believe, as asserted in Mace et al. (2019), that these findings are good evidence that the vast majority of involuntary autobiographical memories experienced in everyday life are a function of semantic-to-autobiographical priming; the result of a multitude of prior information processing. We also believe that because most of these memories occur well after their initial activation (i.e., minutes, hours, or days later), most of them are not functional to the context in which they occur, but are merely the byproducts of semantic-to-autobiographical priming. While there may be studies that suggest that involuntary memories can be functional (Mace \& Atkinson, 2009; Rasmussen, Ramsgaard, \& Berntsen, 2015; but see Kamiya, 2014), the conclusions drawn from such studies are problematic, as they relied on questionnaire methodologies to render judgements about function (see Discussion in Mace, 2018). Perhaps future studies on semantic-to-autobiographical priming will be able to shed more light on this thorny question, as it is important for the science of this area, as well as other similar areas (e.g., involuntary semantic memories, Kvavilashvili \& Mandler, 2004), to determine if involuntary autobiographical memories are functional or the function of other cognitive processes.

If semantic-to-autobiographical priming results in massive amounts of autobiographical memory activations on a daily basis, then one may wonder why we are not inundated with autobiographical memories. We believe that inhibition is a major factor in preventing such memories from coming to mind (e.g., Conway, 2005), though this has yet to be empirically established (see Barzykowski, Radel, Niedzwienska, \& Kvavilashvili, 2019). Research into the question of frequency of involuntary memories has received a fair amount of attention recently, and work in this area is beginning to suggest that a number of factors may prevent widespread inundation of involuntary memories (e.g., cognitive load, cue underload, etc.; for examples, see Barzykowski \& Niedzwienska, 2018a; Barzykowski, et al., 2019; Berntsen, 2009; Vannucci et al., 2015; Vannucci, Pelagatti, Hanczakowski, \& Chiorri, 2019).
Mace et al. (2019) speculated that background activations of autobiographical memories during language processing may be functional in that they may add to our understanding of concepts. This idea was based on the notion in semantic priming that knowledge associated with what one is processing is relevant to such processing (Anderson, 1983). If it is true that autobiographical knowledge is relevant to semantic knowledge, then the results of this study extend this idea beyond language to potentially visual perception. However, we should note that it may be premature to make such claims about semantic-toautobiographical priming, as such priming may occur simply because the autobiographical system is highly sensitive to cues (Conway, 2005). Thus, considerable work will need to be carried out before such functional notions can be confidently extended to semantic-to-autobiographical priming, though we note here the future potential for this line of reasoning.

This study has a number of limitations that should be considered in future work on this topic. Firstly, in the vigilance task some of the target words appeared in the embedded phrases more than once. This may have caused some semantic-to-autobiographical priming in the control group, as some cues may have primed subsequent memories, thus decreasing the magnitude of the priming effect across groups. Secondly, in the sentence-priming group, the sentences may have yielded more secondary targets (e.g., shelf and dusty) than the ones that we identified. Thus, priming on secondary targets for the sentence group may have been a bit higher than our analysis revealed. Thirdly, there may also have been some idiosyncratic processing of the priming stimuli that departed from the central (or intended) meaning of the stimuli (e.g., in the picture group the image for city or home may have been interpreted in different ways). Such differentially processed items may have produced unique semantic-toautobiographical priming, which would not have been detected in our analyses, as they were based on the central, common meaning of primes. This, too, would have contributed to a reduction in the size of the priming effect.

Another limitation concerns low-frequency word primes. As in Mace et al. (2019), we did not obtain priming with lowfrequency words (e.g., garrulous, proclivity, quip) in Experiment 1. Despite this failure, we believe that lowfrequency words also activate autobiographical information, as these items are also likely to be associated with past personal experiences, but often of a form that is not likely to be detected by the priming measure used in this study. That is, low-frequency words are probably associated with fewer autobiographical memories than high-frequency words. In addition, they may be far less likely to be associated with fullblown autobiographical and episodic memories, and many are probably associated with fragmentary autobiographical memories (e.g., a fragment of an episode or other fragmentary autobiographical knowledge). For example, imagine that the item garrulous is incapable of capturing any episodic or 
general autobiographical memories, only fragmentary aspects of the two (e.g., a face or a name from one's past associated with garrulous). These forms of autobiographical knowledge are likely to go undetected with most standard measures of autobiographical memory, including the vigilance task, and therefore our results were insensitive to any such activations (for additional explanations, see Mace et al., 2019). Future studies might be able to address this empirically with different measures of semantic-to-autobiographical priming.

In conclusion, the results of this study have established, once again, that semantic memory activation results in autobiographical memory activation. We have obtained semanticto-autobiographical priming with different stimulus conditions. We have argued that such findings support the general view that semantic-to-autobiographical activations are ubiquitous, occurring whenever semantic access occurs. The results offer the suggestion that certain forms of autobiographical remembering (namely involuntary autobiographical remembering) may be largely non-functional, as such memories may be the mere byproducts of semantic-to-autobiographical priming. Given that involuntary autobiographical memories have received a considerable amount of attention in the literature over the past two decades, future work should be directed towards the question of function as it relates to semantic-toautobiographical priming. As noted above, future work should also investigate semantic-to-autobiographical priming with low-frequency words. Additionally, more work should be done investigating semantic-to-autobiographical priming in visual perception, as well as other areas of perception and information processing in general. Additional positive findings in these areas will strengthen the view that semantic-toautobiographical memory activations are ubiquitous.

Open Practices Statement The data for the experiments reported here are not available and the experiments were not preregistered.

\section{Appendix 1}

Table 3. Words used in the word-priming group

\begin{tabular}{lll}
\hline Music* & Summer* & Cartoons* \\
Friends* & Blatant** & Reading* \\
School* & Lotely** & Proclivity** \\
Quip** & Scruple** & Hiking* \\
Graduation* & Clothing* & Movie* \\
Parade* & Books* & Sports* \\
Art* & Garrulous** & Ruyel** \\
Pensive** & Cell phone* & Car* \\
Doctor* & City* & Exercise* \\
Vacation* & Lilt** & Garden* \\
Home* & Internet* & Mountain* \\
Ostentatious** & Cat* & Beach* \\
Pet* & Betlel** & \\
\hline
\end{tabular}

*Denotes a high-frequency word

**Denotes a low-frequency word or non-word

\section{Appendix 2}

Table 4. Sentences used in the sentence-priming group

People enjoy listening to music.

Joe's shows great proclivity.

Mary colored the wall in a blatant grey.

Her friends go paintballing each weekend.

The school just got repainted with its colors and mascot.

The parade dragged on for hours.

Art is part of John's everyday life.

The doctor wrote a prescription.

The students had to scruple to class.

It rained throughout Mary's entire vacation

He just bought his first home.

The table had a small lilt to the right.
She never had a pet growing up, but she wants She is very allergic to cats. one now.

Joe's summer ended too soon.

Yesterday he rolled through a ruyel.

The list was organized in a lotely manner.

Her clothing got soaked during the rainstorm last night.

The lightning came down with great betlel.

Their outfits that day were very quip.

He threw the rock with such garrulous force.

The books on this shelf are dusty.

The cell phone dropped in the sink.

The city is loud and congested.

Joe has always been an ostentatious wall flower.

Mary finds cartoons to be a great way to unwind after a long day.

He reads in bed before falling asleep each night.

The deviled eggs tasted very pensive.

Hiking through the woods relaxed her.

The movie had a predictable ending.

Joe's car overheated during a road trip.

Jim likes sports.

Mary likes to exercise.

Jim likes working in his garden.

She went to the mountain.

He does most of his research and work on the Most people like the beach. internet.

Note: Underlined words were primary targets or foils used in the word-priming group; words in italics are additional concepts that served as secondary targets 


\section{References}

Anderson, J. R. (1983). A spreading activation theory of memory. Journal of Verbal Learning and Verbal Behavior, 22, 261-295.

Ball, C.T., \& Hennessey, J. (2009). Subliminal priming of autobiographical Memories. Memory, 17, 311-322.

Barzykowski, K., \& Niedzwienska, A., (2018a). Involuntary autobiographical memories are more often reported during high cognitive load tasks. Acta Psychologica, 182, 119-128.

Barzykowski, K., \& Niedzwienska, A., (2018b). Priming involuntary autobiographical memories in the lab. Memory, 26, 277-289.

Barzykowski, K., Radel, R., Niedzwienska, A., \& Kvavilashvili, L. (2019). Why are we not flooded by involuntary thoughts about the past and future? Testing the cognitive inhibition dependency hypothesis. Psychological Research, 83, 666-683.

Barzykowski, K., \& Staugaard, S.R., (2016). Does retrieval intentionality really matter? Similarities and differences between involuntary memories and directly and generatively retrieved voluntary memories. British Journal Psychology, 107, 519-536.

Berntsen, D. (2009). Involuntary autobiographical memories: An introduction to the unbidden past. Cambridge: Cambridge University Press.

Coane, J.H., \& Balota, D.A. (2009). Priming the holiday spirit: Persistent activation due to extra-experimental experiences. Psychonomic Bulletin \& Review, 16, 1124-1128.

Collins, A. M., \& Loftus, E. F. (1975). A spreading-activation theory of semantic processing. Psychological Review, 82, 407-428.

Conway, M. A. (1990). Associations between autobiographical memories and concepts. Journal of Experimental Psychology: Learning, Memory, and Cognition, 16, 799-812.

Conway, M.A. (2001). Sensory-perceptual episodic memory and its context: Autobiographical memory. In A. Baddeley, J.P. Aggleton, \& M.A. Conway (Eds.), Episodic memory: New directions in research (pp. 53-70). New York: Oxford University Press.

Conway, M. A. (2005). Memory and the self. Journal of Memory and Language, 53, 594-628.

Conway, M.A., \& Bekerian, D.A. (1987). Organization in autobiographical memory. Memory \& Cognition, 15, 119-132.

Fitzgerald, J.M. (1996). Intersecting meanings of reminiscence in adult development and ageing. In D.C. Rubin (Ed.), Remembering our past: Studies in autobiographical memory (pp. 360-383). New York: Cambridge University Press.

Kamiya, S. (2014). Relationship between frequency of involuntary autobiographical memories and cognitive failures. Memory. 22, 839851.

Kvavilashvili, L., \& Mandler, G. (2004). Out of one's mind: A study of involuntary semantic memories. Cognitive Psychology, 48, 47-94.

Mace, J.H. (2005). Priming involuntary autobiographical memories. Memory, 13, 874-884.
Mace, J.H. (2007). Involuntary memory. Malden, MA: Blackwell Publishing.

Mace, J.H. (2010). Understanding autobiographical remembering from a spreading activation perspective. In J.H. Mace (Ed.), The act of remembering: Toward an understanding of how we recall the past (pp. 43-55). Malden, MA: Wiley-Blackwell.

Mace, J.H. (2018). Involuntary autobiographical memories: Spontaneous recollections of the past. In K.C.R. Fox, \& K. Christoff (Eds.), The Oxford Handbook of spontaneous thought: Mind-wandering, dreaming, creativity, and clinical disorders, Oxford University Press.

Mace, J.H., \& Atkinson, E. (2009). Can we determine the functions of everyday involuntary autobiographical memories? In M. Kelly (Ed.), Applied Memory, Nova Science Publishers.

Mace, J.H., \& Clevinger, A.M. (2013). Priming voluntary autobiographical memories: Implications for the organization of autobiographical memory and voluntary recall processes. Memory, 21, 524-536.

Mace, J.H., McQueen, Hayslett, K.E., Staley, B. A, \& Welch, T.J. (2019). Semantic memories prime autobiographical memories: Implications for everyday autobiographical remembering. Memory \& Cognition, 47, 299-312.

McNamara, T. P. (1992). Theories of priming: I. Associative distance and lag. Journal of Experimental Psychology: Learning, Memory, and Cognition, 18, 1173-1190.

McNamara, T. P. (2005). Semantic priming: Perspectives from memory and word recognition. New York: Psychology Press.

Rasmussen, A.S., Ramsgaard, \& Berntsen, D. (2015). The frequency and functions of involuntary and voluntary autobiographical memories across the day. The Psychology of Consciousness: Theory, Research and Practice, 2, 185-205.

Roediger, H.L.,III, \& McDermott, K. (1993). Implicit memory in normal human participants. In F. Boller \& J. Grafman (Eds.), Handbook of neuropsychology (Vol. 8), pp. 63-131. New York: Elsevier Science.

Schlagman, S., \& Kvavilashvili, L. (2008). Involuntary autobiographical memories in and outside the laboratory: How different are they from voluntary autobiographical memories? Memory \& Cognition, 36, 920-932.

Vannucci, M., Pelagatti, C., Hanczakowski, M. G., \& Chiorri C. (2019). Visual attention load affects the frequency of involuntary autobiographical memories and their level of meta-awareness. Memory \& Cognition, 47, 117-129.

Vannucci, M., Pelagatti, C., Hanczakowski, M., Mazzoni, G., \& Paccani, C.R. (2015). Why are we not flooded by involuntary autobiographical memories? Few cues are more effective than many. Psychological Research, 79, 1077-1085.

Publisher's note Springer Nature remains neutral with regard to jurisdictional claims in published maps and institutional affiliations. 